\title{
The Synthesis and Inhibitory Activity of Dethiotrypanothione and Analogues Against Trypanothione Reductase
}

\author{
Josephine A. Czechowicz, April K. Wilhelm, Maroya D. Spalding, Anna M. Larson, Linnea K. \\ Engel, and David G. Alberg \\ Department of Chemistry, Carleton College, Northfield, Minnesota 55057
}

\section{Abstract}

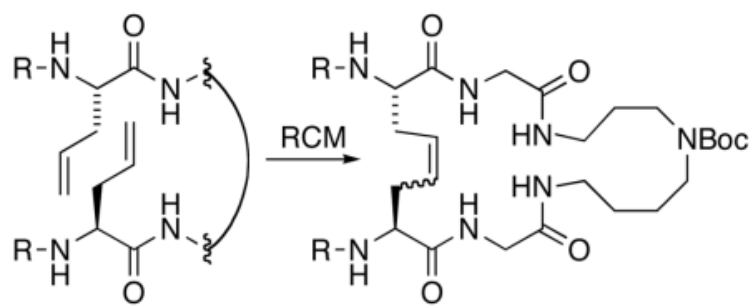

Trypanothione reductase (TR) catalyzes the NADPH-dependent reduction of trypanothione disulfide (1). TR plays a central role in the trypanosomatid parasite's defense against oxidative stress and has emerged as a promising target for antitrypanosomal drugs. We describe the synthesis and activity of dethiotrypanothione and analogues (2-4) as inhibitors of T. cruzi TR. The syntheses of these macrocycles feature ring-closing olefin metathesis $(\mathrm{RCM})$ reactions catalyzed by ruthenium catalyst 17. Derivative 4 is our most potent inhibitor with a $K_{i}=16 \mu \mathrm{M}$.

The trypanosomatid parasites are the causative agents of several tropical diseases in both humans and domestic animals. Examples include Chagas' disease (Trpanosoma cruzi), African sleeping sickness (T. brucei gambiense and T. b. rhodensiense, West African and East African trypanosomiasis, respectively), and nagana cattle disease (T. b. brucei, T. conglense). ${ }^{1}$ These parasites have a unique thiol metabolism centered on the NADPH-dependent flavoenzyme trypanothione reductase (TR), which catalyzes the reduction of the antioxidant trypanothione ${ }^{2}\left(\mathbf{1}, N^{1}, N^{8}\right.$-bis(glutathionyl)spermidine) from its disulfide to its dithiol form. ${ }^{3}$ The parasites rely on the trypanothione/TR system to maintain an intracellular reducing environment and to combat oxidative stress, ${ }^{4}$ and several genetic studies have demonstrated the parasites' dependence on TR for growth and virulence. 5

In the trypanosomatids, the trypanothione/TR couple replaces the closely related glutathione/ glutathione reductase (GR) system found in host organisms. ${ }^{3}$ TR and GR display a high degree of sequence ${ }^{3}$ and structural homology, ${ }^{6}$ the catalytic mechanisms of both enzymes are essentially identical, ${ }^{7}$ and yet the enzymes display almost complete specificity for their respective substrates. ${ }^{8}$ This mutually exclusive substrate specificity between the parasite and host enzymes, and the parasite's sensitivity to a loss of TR function, combine to make TR a promising drug target. Accordingly, a large number of TR inhibitors have been reported. 4,9 
<smiles>[X][X]C(CNC(=O)CNC(=O)CNC(=O)CNC(=O)[C@H](C[X])N[R])C(=O)N[R]</smiles>

1: $R=\gamma$-glutamyl, $X=S$ (trypanothione disulfide)

2: $R=\gamma$-glutamyl, $X=\mathrm{CH}_{2}$ (dethiotrypanothione)

3: $\mathrm{R}=\mathrm{Cbz}, \mathrm{X}=\mathrm{CH}_{2}$

4: $\mathrm{R}=\mathrm{Fmoc}, \mathrm{X}=\mathrm{CH}_{2}$

Here we report the synthesis and inhibitory activity of $\mathbf{2 - 4}$, structural analogues of thenatural substrate. Compound 2, which we have named dethiotrypanothione, contains all of the structural elements of trypanothione, except that the substrate's redox-active disulfide moiety has been replaced by a pair of methylene groups. Analogues $\mathbf{3}$ and $\mathbf{4}$ also lack the disulfide and, in addition, trypanothione's $\gamma$-glutamyl groups have been replaced by hydrophobic aromatic moieties in these compounds. We prepared these analogues to explore the curious tolerance the enzyme displays for substrate analogues incorporating Cbz groups and other hydrophobic moieties in place of the zwitterionic glutamate residues. ${ }^{10}$

Our syntheses of dethiotrypanothione and analogues feature ring-closing olefin metathesis (RCM) reactions catalyzed by second-generation Grubb's catalyst $\mathbf{1 7},{ }^{11}$ as shown in Scheme 1. RCM has emerged as a powerful tool in the formation of macrocyclic compounds due to the exceptional chemoselectivity of the recently developed catalysts (such as 17) and their tolerance of diverse functionality. ${ }^{12} \mathrm{RCM}$ seemed particularly well suited to the synthesis of our 24-membered ring macrocyles $\mathbf{2 - 4}$, since we envisioned that the required RCM substrates could be efficiently constructed from spermidine and commercially available amino acid derivatives, in a bidirectional fashion from common intermediate 7 .

The known diamine intermediate $\mathbf{7}$ derived from its Cbz-protected derivative $\mathbf{6}$, which was first reported by Henderson and coworkers in their synthesis of trypanothione. ${ }^{13}$ Their fourstep method afforded $\mathbf{6}$ in about 50\% overall yield and proceeded through a hexahydropyrimidine derivative of spermidine developed by Ganem, et al. 14 which elegantly distinguishes the three amino groups of spermidine. We found that $\mathbf{6}$ is more efficiently prepared by direct treatment of spermidine with two equivalents of Cbz-glycine $\mathrm{N}$ hydroxysuccinimide ester. This reaction proceeded with selective acylation of the primary amines without detectable modification of spermidine's secondary nitrogen. ${ }^{15}$ Subsequent blocking of the secondary amine with the Boc group provided $\mathbf{6}$ in just two steps from spermidine, with an $80 \%$ overall yield.

Hydrogenolysis of the Cbz groups of 6 followed by PyBop-mediated ${ }^{16}$ coupling of the resulting diamine 7 with two equivalents of either Cbz-allylglycine or Fmoc-allylglycine provided $\mathbf{8}$ or $\mathbf{9}$, respectively. Intermediates $\mathbf{8}$ and $\mathbf{9}$ were the substrates for the RCM reactions in the syntheses of inhibitors $\mathbf{3}$ and $\mathbf{4}$, respectively. In the case of the synthesis of $\mathbf{2 , 9}$ was elaborated to the bis- $\gamma$-glutamyl RCM substrate 10, using DEPBT ${ }^{17}$ as the peptide coupling reagent. The RCM reactions of $\mathbf{8 - 1 0}$ were carried out under high dilution in refluxing $\mathrm{CH}_{2} \mathrm{Cl}_{2}$, in the presence of 27-35 mole-percent of catalyst 17,11 with yields ranging from 5592\%. Olefins 11-13 (E/Z-mixtures) were reduced to their saturated derivatives 14-16 and, 
finally, the $t$-butyl-based protecting groups of $\mathbf{1 4}, \mathbf{1 5}$, and $\mathbf{1 6}$ were removed under acidic conditions to afford inhibitors $3, \mathbf{4}$, and $\mathbf{2}$, respectively.

Trypanothione analogues $\mathbf{2 - 4}$ were evaluated as reversible inhibitors of recombinant $T$. cruzi TR. ${ }^{18}$ TR activity was assayed using disulfide substrate $\mathbf{1 8}$, developed by Douglas and coworkers, as an effective alternative to the expensive trypanothione. ${ }^{19}$ The reaction was monitored spectrophotometrically by following the oxidation of NADPH at $340 \mathrm{~nm} .{ }^{20}$ The Ki values for each inhibitor are given in Table 1.<smiles>[X][C]C(N[R])C(=O)NCC(=O)NCCCN(C)C</smiles>
18: $\mathrm{R}=\mathrm{Cbz}, \mathrm{X}=\mathrm{S}$
19: $R=\gamma$-glutamyl, $X=S$
20: $\mathrm{R}=\mathrm{Cbz}, \mathrm{X}=\mathrm{CH}_{2}$

Dethiotrypanothione proved to be a very poor inhibitor of the enzyme. On the otherhand, replacing the charged $\gamma$-glutamyl moieties of $\mathbf{2}$ with Cbz groups $(\mathbf{3})$ resulted in nearly 6-fold stronger binding, while 4, with the larger Fmoc groups in place of the $\gamma$-glutamyl moieties, was our most potent inhibitor. A thorough analysis of the inhibitory activity of $\mathbf{4}$ was precluded by its low solubility under the assay conditions, restricting the concentration range over which we could evaluate this compound. Thus, our determination of a $\mathrm{K}_{\mathrm{i}}=16 \mu \mathrm{M}$ for $\mathbf{4}$ was based onassays using just two inhibitor concentrations ( 6.4 and $11.1 \mu \mathrm{M})$, each examined over a range off our substrate concentrations. ${ }^{21}$ Since we were not able to assay $\mathbf{4}$ at concentrations above its determined $\mathrm{K}_{\mathrm{i}}$ value, the precise value of $16 \mu \mathrm{M}$ should be read with some caution.

Nevertheless, it is clear that the Fmoc derivative binds TR with an affinity of at least an order of magnitude greater than that of $\mathrm{Cbz}$ derivative $\mathbf{3}$.

We also tested our inhibitors for activity against yeast GR. For compounds $\mathbf{3}$ and $\mathbf{4}$, no inhibition of GR was observed up to concentrations of $1.1 \mathrm{mM}$ of 3 and $16 \mu \mathrm{M}$ of 4 (the solubility limit for this compound). Dethiotrypanothione (2) at $5 \mathrm{mM}$ showed no detectable inhibition of GR; however, at $10 \mathrm{mM} 2$ we did observe about $20 \%$ inhibition compared to control assays, indicating that $\mathbf{2}$ does interact with GR at very high concentrations. Our lack of sufficient quantities of $\mathbf{2}$ precluded a more complete analysis of dethiotrypanothione's activity against GR. Nevertheless, all three inhibitors displayed decided selectivity for the parasite enzyme.

Given the structural similarity between dethiotrypanothione 2 and trypanothione disulfide $\left(\mathrm{K}_{\mathrm{m}}=50 \mu \mathrm{M}, \mathrm{k}_{\mathrm{cat}}=192 \mathrm{~s}^{-1}\right),{ }^{18}$ we did not expect 2 to be such a poor TR ligand. Of course, 2-4 do lack the substrate's disulfide group and the potential electrostatic binding contacts that this moiety may afford. Furthermore, the geometry of the disulfide moiety, which typically exhibits about a $90^{\circ}$ dihedral angle about the $\mathrm{S}-\mathrm{S}$ bond, would be expected to be quite different 
than the geometry of the methylene bridge in our inhibitors. We have previously reported the TR inhibitory activity of $\mathbf{2 0}$, an inhibitor that also lacks the disulfide moiety. ${ }^{10 \mathrm{c}}$ Inhibitor $\mathbf{2 0}$ $\left(\mathrm{K}_{\mathrm{i}}=30 \mu \mathrm{M}\right)$ was modeled after TR substrate $\mathbf{1 8}\left(\mathrm{K}_{\mathrm{m}}=6.7 \mu \mathrm{M}\right)$. Interestingly, like 2 and trypanothione disulfide, $\mathbf{2 0}$ and $\mathbf{1 8}$ also differ only in the substitution of the disulfide group by a pair of methylenes, yet unlike $\mathbf{2}$ and trypanothione disulfide, the affinities of $\mathbf{2 0}$ and $\mathbf{1 8}$ for TR appear to be comparable.

We were not surprised by the affinity TR displayed for analogues $\mathbf{3}$ and $\mathbf{4}$, since it is well established that TR tolerates hydrophobic groups in the place of the $\gamma$-glutamyl residues in alternate substrates such as $18 .{ }^{10}$ Douglas and coworkers explored the enzyme's tolerance for $\gamma$-glutamyl-modified substrates in their study comparing the activities of derivatives based on $\gamma$-glutamylcysteinylglycyl-3-dimethylaminopropylamide (19). ${ }^{10 \mathrm{a}}$ While the Cbz-modified derivative 18 retains only $58 \%$ of the catalytic efficiency $\left(\mathrm{k}_{\mathrm{cat}} / \mathrm{K}_{\mathrm{m}}\right)$ observed for $\mathbf{1 9}, \mathbf{1 8}$ does have a significantly lower $\mathrm{K}_{\mathrm{m}}$ than $19\left(52 \mu \mathrm{M}\right.$ for 18 versus $225 \mu \mathrm{M}$ for 19), ${ }^{22}$ suggesting stronger binding of the Cbz groups compared to the $\gamma$-glutamyl residues. Based on crystallographic data, Douglas' group proposed that a Cbz moiety binds in a hydrophobic pocket (the 'Z-site') on the enzyme that is not utilized in the binding of the natural substrate. $9 c, 23$ The more potent inhibition observed for our Fmoc derivative $\mathbf{4}$ suggests that this putative $\mathrm{Z}$-site may favorably accommodate aromatic groups that are significantly larger than the $\mathrm{Cbz}$ moiety. On the other hand, in addition to the Z-site, the enzyme also possesses a hydrophobic patch comprised of the side chains of Leu18, Trp22, Tyr111, and Met114, where trypanothione's spermidine moiety binds. ${ }^{6}$ The aromatic acridine ring of a quinacrine-based inhibitor was recently shown to bind in this hydrophobic site, ${ }^{24}$ and this pocket is also the putative binding site of a number of related hydrophobic tricyclic TR inhibitors. ${ }^{25}$ It seems unlikely, but it is possible that $\mathbf{3}$ and $\mathbf{4}$ are turned around in the active site, binding in an orientation that places their aromatic moieties in this hydrophobic site that normally binds the substrate's spermidine moiety.

In conclusion we have developed syntheses of dethiotrypanothione and related trypanothione analogues featuring RCM macrocyclizations. Dethiotrypanothione proved to be a very poor inhibitor of TR despite its close structural similarity to the natural substrate. However, the more hydrophobic analogues $\mathbf{3}$ and $\mathbf{4}$ are more effective inhibitors, underscoring the enzyme's general affinity for hydrophobic ligands.

\section{Experimental Section}

\section{Dethiotrypanothione (2)}

${ }^{1} \mathrm{H}$ NMR (400 MHz, CD $\left.{ }_{3} \mathrm{OD}\right) \delta 4.26(\mathrm{dd}, 1 \mathrm{H}, \mathrm{J}=6.8,6.8 \mathrm{~Hz}), 4.25(\mathrm{dd}, 1 \mathrm{H}, \mathrm{J}=5.9,8.5 \mathrm{~Hz})$, $4.04(\mathrm{dd}, 1 \mathrm{H}, \mathrm{J}=6.5,6.5 \mathrm{~Hz}), 4.03(\mathrm{dd}, 1 \mathrm{H}, \mathrm{J}=6.3,6.3 \mathrm{~Hz}), 3.88(\mathrm{~d}, 1 \mathrm{H}, \mathrm{J}=16.1 \mathrm{~Hz}), 3.86(\mathrm{~d}$, $1 \mathrm{H}, \mathrm{J}=16.1 \mathrm{~Hz}), 3.77(\mathrm{~d}, 1 \mathrm{H}, \mathrm{J}=16.1 \mathrm{~Hz}), 3.73(\mathrm{~d}, 1 \mathrm{H}, \mathrm{J}=16.1 \mathrm{~Hz}), 3.40-3.33(\mathrm{~m}, 2 \mathrm{H}), 3.32-3.24$ $(\mathrm{m}, 2 \mathrm{H}), 3.03(\mathrm{t}, 2 \mathrm{H}, \mathrm{J}=6.8 \mathrm{~Hz}), 3.00(\mathrm{t}, 2 \mathrm{H}, \mathrm{J}=7.2 \mathrm{~Hz}), 2.60-2.53(\mathrm{~m}, 4 \mathrm{H}), 2.29-2.10(\mathrm{~m}, 4 \mathrm{H})$, 1.93-1.84 (m, 2H), 1.84-1.66 (m, 6H), 1.65-1.57 (m, 2H), 1.51-1.35 (m, 2H) ppm; ${ }^{13} \mathrm{C}$ NMR (100 MHz, $\left.\mathrm{CD}_{3} \mathrm{OD}\right) \delta 175.2,175.0,174.6,174.5,172.8,171.9,171.6,55.0,53.5,45.7,44.0$, 43.8, 39.0, 36.5, 32.1, 27.3, 27.1, 26.9, 26.1, 25.7, 23.9 ppm; HRMS (ESI) $\mathrm{m} / \mathrm{z}$ calcd for $\mathrm{C}_{29} \mathrm{H}_{52} \mathrm{~N}_{9} \mathrm{O}_{10}[\mathrm{M}+\mathrm{H}]^{+}$686.3832, found 686.3831 .

\section{Inhibitor 3}

${ }^{1} \mathrm{H}$ NMR (400 MHz, $15 \% \mathrm{CH}_{3} \mathrm{OD} / \mathrm{CDCl}_{3}$ with a trace of TFA- $d$ added) $\delta 7.39-7.28(\mathrm{~m}, 10 \mathrm{H})$, 5.15-5.05 (m, 2H), 4.17-4.09 (m, 2H), 3.90-3.72 (m, 4H), 3.52-3.11 (m, 4H), 3.00-2.84 (m, $4 \mathrm{H}), 1.94-1.83(\mathrm{~m}, 2 \mathrm{H}), 1.83-1.62(\mathrm{~m}, 6 \mathrm{H}), 1.62-1.51(\mathrm{~m}, 2 \mathrm{H}), 1.48-1.31(\mathrm{~m}, 4 \mathrm{H}) \mathrm{ppm} ;{ }^{13} \mathrm{C}$ NMR $\left(100 \mathrm{MHz}, 15 \% \mathrm{CD}_{3} \mathrm{OD} / \mathrm{CDCl}_{3}\right.$ with a trace of TFA added) $\delta 174.7(\mathrm{C}), 174.6(\mathrm{C}), 172.0$ $(\mathrm{C}), 170.9(\mathrm{C}), 157.62(\mathrm{C}), 157.56(\mathrm{C}), 136.9(\mathrm{C}), 129.0(\mathrm{CH}), 128.6(\mathrm{CH}), 128.2(\mathrm{CH}), 67.4$ 
$\left(\mathrm{CH}_{2}\right)$, 55.4(CH), 55.3(CH), 47.7 $\left(\mathrm{CH}_{2}\right), 45.0\left(\mathrm{CH}_{2}\right), 43.5\left(\mathrm{CH}_{2}\right), 43.3\left(\mathrm{CH}_{2}\right), 38.3\left(\mathrm{CH}_{2}\right), 35.8$ $\left(\mathrm{CH}_{2}\right), 31.7\left(\mathrm{CH}_{2}\right), 30.1\left(\mathrm{CH}_{2}\right), 26.5\left(\mathrm{CH}_{2}\right), 26.2\left(\mathrm{CH}_{2}\right), 25.0\left(\mathrm{CH}_{2}\right), 24.6\left(\mathrm{CH}_{2}\right), 23.1\left(\mathrm{CH}_{2}\right) \mathrm{ppm}$; HRMS (ESI) $\mathrm{m} / z$ calcd for $\mathrm{C}_{35} \mathrm{H}_{50} \mathrm{~N}_{7} \mathrm{O}_{8}[\mathrm{M}+\mathrm{H}]^{+} 696.3721$, found $696.3738 ; \mathrm{m} / z$ calcd for $\mathrm{C}_{35} \mathrm{H}_{49} \mathrm{~N}_{7} \mathrm{NaO}_{8}[\mathrm{M}+\mathrm{Na}]^{+}$718.3541, found 718.3557 .

\section{Inhibitor 4}

${ }^{1} \mathrm{H}$ NMR $\left(400 \mathrm{MHz}, 10 \% \mathrm{CD}_{3} \mathrm{OD} / \mathrm{CDCl}_{3}\right.$ with ca. $3 \%$ TFA) $\delta 7.76(\mathrm{~d}, 4 \mathrm{H}, \mathrm{J}=7.3 \mathrm{~Hz}), 7.62$ (d, 4H, J=6.6 Hz), $7.40(\mathrm{t}, 4 \mathrm{H}, \mathrm{J}=7.05 \mathrm{~Hz}), 7.31(\mathrm{t}, 4 \mathrm{H}, \mathrm{J}=6.8 \mathrm{~Hz}), 4.52-4.33(\mathrm{~m}, 4 \mathrm{H}), 4.25-4.17$ $(\mathrm{m}, 2 \mathrm{H}), 4.15-4.08(\mathrm{~m}, 2 \mathrm{H}), 3.90-3.77(\mathrm{~m}, 4 \mathrm{H}), 3.47-3.13(\mathrm{~m}, 4 \mathrm{H}), 3.01-2.83(\mathrm{~m}, 4 \mathrm{H}), 1.93-1.49$ $(\mathrm{m}, 8 \mathrm{H}), 1.45-1.16(\mathrm{~m}, 6 \mathrm{H}) \mathrm{ppm} ;{ }^{13} \mathrm{C}$ NMR $\left(100 \mathrm{MHz}, 10 \% \mathrm{CD}_{3} \mathrm{OD} / \mathrm{CDCl}_{3}\right.$ with ca. $1-2 \%$ TFA) $\delta 173.9,173.8,171.3,170.2,161.5,156.7,156.7,143.4,143.4,141.0,127.4,126.7$, 124.61, 124.57. 119.6, 77.2, 66.6, 54.5, 46.9, 46.8, 44.1, 42.7, 42.5, 37.5, 35.0, 30.9, 29.3, 25.6, 25.5, 24.1, 23.8, 22.2 ppm; HRMS (ESI) $m / z$ calcd for $\mathrm{C}_{49} \mathrm{H}_{58} \mathrm{~N}_{7} \mathrm{O}_{8}[\mathrm{M}+\mathrm{H}]^{+}$872.4341, found 872.4317.

\section{$\boldsymbol{N}^{\mathbf{1}}, \boldsymbol{N}^{\mathbf{8}}$-Bis(benzyloxycarbonylglycyl)spermidine (5)}

m.p. $148-149{ }^{\circ} \mathrm{C}\left(\mathrm{lit} .13149-150{ }^{\circ} \mathrm{C}\right) ;{ }^{1} \mathrm{H}$ NMR $\left(400 \mathrm{MHz}\right.$, DMSO- $\left.d_{6}\right) \delta 7.90(\mathrm{t}, 1 \mathrm{H}, \mathrm{J}=5.4$ $\mathrm{Hz}), 7.86(\mathrm{t}, 1 \mathrm{H}, \mathrm{J}=5.7 \mathrm{~Hz}), 7.45-7.30(\mathrm{~m}, 11.6 \mathrm{H}), 7.09-7.02(\mathrm{~m}, 0.4 \mathrm{H} \mathrm{CONH}$, minor rotomer), $5.03(\mathrm{~s}, 4 \mathrm{H}), 3.57(\mathrm{~d}, 4 \mathrm{H}, \mathrm{J}=6.0 \mathrm{~Hz}), 3.09(\mathrm{dt}, 2 \mathrm{H}, \mathrm{J}=6.4,6.4 \mathrm{~Hz}), 3.05(\mathrm{dt}, 2 \mathrm{H}, \mathrm{J}=6.2,6.2)$, 2.48-2.43 (m, 4H), 1.52 (quin, 2H, J=6.7 Hz), 1.44-1.33 (m, 4H); 13C NMR (100 MHz, DMSO$\left.d_{6}\right) \delta 168.8,168.7,156.4,137.0,136.9,128.2,127.7,127.6,65.4,48.8,46.7,43.5,38.4,36.9$, 29.2, 26.9, $26.7 \mathrm{ppm}$; LRMS (ESI) $\mathrm{m} / z 528.4[\mathrm{M}+\mathrm{H}]^{+}, 550.3[\mathrm{M}+\mathrm{Na}]^{+}$.

\section{$\boldsymbol{N}^{\mathbf{1}}, \boldsymbol{N}^{\mathbf{8}}$-Bis(benzyloxycarbonylglycyl)- $\boldsymbol{N}^{\mathbf{4}}$-t-butoxycarbonylspermidine (6)}

${ }^{1} \mathrm{H} \mathrm{NMR}\left(400 \mathrm{MHz}, \mathrm{CDCl}_{3}\right) \delta$ 7.36-7.25 (m, 10.7H), 6.93-6.70 (m, 1.3H), 6.14-5.90 (m, 2H), $5.09(\mathrm{~s}, 2 \mathrm{H}), 5.08(\mathrm{~s}, 2 \mathrm{H}), 3.84-3.81(\mathrm{~m}, 4 \mathrm{H}), 3.26-3.05(\mathrm{~m}, 8 \mathrm{H}), 1.71-1.56(\mathrm{~m}, 2 \mathrm{H}), 1.54-1.39$ (m, 4H), 1.42 (s, 9H) ppm; 13C NMR (100 MHz, $\left.\mathrm{CDCl}_{3}\right) \delta 169.4(\mathrm{C}), 156.8(\mathrm{C}), 156.1(\mathrm{C})$, 136.3(C), 136.2(C), 128.51(CH), 128.47(CH), 128.2(CH), 128.1(CH), 128.0(CH), 79.7(C), 67.0 $\left(\mathrm{CH}_{2}\right), 66.8\left(\mathrm{CH}_{2}\right), 46.8\left(\mathrm{CH}_{2}\right), 45.1\left(\mathrm{CH}_{2}\right), 44.5\left(\mathrm{CH}_{2}\right), 43.9\left(\mathrm{CH}_{2}\right), 38.8\left(\mathrm{CH}_{2}\right), 36.2\left(\mathrm{CH}_{2}\right)$, 29.3 $\left(\mathrm{CH}_{3}, t\right.$-butyl, minor rotamer), 28.4( $\left(\mathrm{CH}_{3}\right), 27.8\left(\mathrm{CH}_{2}\right), 26.7\left(\mathrm{CH}_{2}\right), 25.8\left(\mathrm{CH}_{2}\right)$ ppm; LRMS (ESI) $\mathrm{m} / 2650.3(\mathrm{M}+\mathrm{Na})^{+}$.

\section{Diene 8}

${ }^{1} \mathrm{H}$ NMR (400 MHz, $\mathrm{CDCl}_{3}$, $) \delta$ 7.73-6.87 (m, 14H), 6.06-5.62 (m, 4H), 5.19-4.98 (m, 8H), 4.40-4.16 (m, 2H), 4.10-3.66 (m, 4H), 3.36-3.00 (m, 8H), 2.69-2.34 (m, 4H), 1.77-1.60 (m, $2 \mathrm{H}), 1.59-1.33\left(\mathrm{~m}, 13 \mathrm{H}\right.$, within this multiplet lies the t-butyl group $\mathrm{CH}_{3}$ singlet at $\left.1.42 \mathrm{ppm}\right)$ ppm; ${ }^{13} \mathrm{C} \mathrm{NMR}\left(100 \mathrm{MHz}, \mathrm{CDCl}_{3}\right) \delta$ 172.1(C), 169.0(C), 156.5(C), 156.1(C), 136.1(C), 136.0 $(\mathrm{C}), 133.0(\mathrm{CH}), 132.7(\mathrm{CH}), 128.6(\mathrm{CH}), 128.5(\mathrm{CH}), 128.3(\mathrm{CH}), 128.2(\mathrm{CH}), 128.0(\mathrm{CH}), 119.2$ $\left(\mathrm{CH}_{2}\right), 119.0\left(\mathrm{CH}_{2}\right), 79.7(\mathrm{C}), 67.13\left(\mathrm{CH}_{2}\right), 67.10\left(\mathrm{CH}_{2}\right), 54.7(\mathrm{CH}), 47.0\left(\mathrm{CH}_{2}\right), 45.3\left(\mathrm{CH}_{2}\right), 44.1$ $\left(\mathrm{CH}_{2}\right)$, 43.2( $\left.\mathrm{CH}_{2}\right), 38.7\left(\mathrm{CH}_{2}\right), 36.6\left(\mathrm{CH}_{2}\right), 28.4\left(\mathrm{CH}_{3}\right), 27.8\left(\mathrm{CH}_{2}\right), 26.6\left(\mathrm{CH}_{2}\right), 25.9\left(\mathrm{CH}_{2}\right)$; HRMS (ESI) $\mathrm{m} / z$ calcd for $\mathrm{C}_{42} \mathrm{H}_{59} \mathrm{~N}_{7} \mathrm{NaO}_{10}[\mathrm{M}+\mathrm{Na}]^{+}$844.4227, found 844.4217.

\section{Diene 9}

${ }^{1} \mathrm{H}$ NMR $\left(400 \mathrm{MHz}\right.$, acetone- $\left.d_{6}\right) \delta$ 7.91-7.83 (m, 2H CON$\left.\underline{\mathrm{H}}\right), 7.86$ (d, 4H, J=7.6 Hz), 7.69 (dd, $4 \mathrm{H}, \mathrm{J}=3.8,7.5 \mathrm{~Hz}), 7.41(\mathrm{t}, 4 \mathrm{H}, \mathrm{J}=7.5 \mathrm{~Hz}), 7.41-7.24(\mathrm{~m}, 2 \mathrm{H}, \mathrm{N} \underline{\mathrm{H}}), 7.32(\mathrm{t}, 4 \mathrm{H}, \mathrm{J}=7.6 \mathrm{~Hz}), 6.95$ (d, 2H, J=7.0 Hz), 5.85 (dddd, 2H, J=7.0, 7.0, 10.2, $17.1 \mathrm{~Hz}$ ), 5.15 (dddd, 2H, J=1.4, 1.4, 3.2, $17.1 \mathrm{~Hz}$ ), 5.06 (dddd, $2 \mathrm{H}, \mathrm{J}=1.0,2.1,3.2,10.2 \mathrm{~Hz}), 4.43-4.35(\mathrm{~m}, 2 \mathrm{H}), 4.35-4.28(\mathrm{~m}, 2 \mathrm{H})$, 4.26-4.17 (m, 4H), $3.92(\mathrm{dd}, 2 \mathrm{H}, \mathrm{J}=6.0,16.7 \mathrm{~Hz}), 3.82-3.73(\mathrm{~m}, 2 \mathrm{H}), 3.24-3.07(\mathrm{~m}, 8 \mathrm{H})$, 2.69-2.58 (m, 2H), 2.53-2.43 (m, 2H), 1.74-1.59 (m, 2H), 1.52-1.40 (m, 4H), $1.39(\mathrm{~s}, 9 \mathrm{H})$ ppm; ${ }^{13} \mathrm{C}$ NMR (100 MHz, acetone- $\left.d_{6}\right) \delta 172.7(\mathrm{C}), 169.7(\mathrm{C}), 157.4(\mathrm{C}), 156.2(\mathrm{C}), 144.9(\mathrm{C})$, 144.8(C), 142.0(C), 135.0(CH), 134.9(CH), 128.5(CH), 127.9(CH), 126.1(CH), 120.7(CH), 
118.3 $\left(\mathrm{CH}_{2}\right), 79.3(\mathrm{C}), 67.4\left(\mathrm{CH}_{2}\right), 56.0(\mathrm{CH}), 55.5\left(\mathrm{CH}_{2}\right), 47.9(\mathrm{CH}), 47.4\left(\mathrm{CH}_{2}\right), 45.0\left(\mathrm{CH}_{2}\right), 43.6$ $\left(\mathrm{CH}_{2}\right), 43.5\left(\mathrm{CH}_{2}\right), 39.3\left(\mathrm{CH}_{2}\right), 36.9\left(\mathrm{CH}_{2}\right), 32.0\left(\mathrm{CH}_{3}\right), 28.6\left(\mathrm{CH}_{3}\right), 26.54\left(\mathrm{CH}_{2}\right) \mathrm{ppm}$; HRMS (ESI) $\mathrm{m} / z$ calcd for $\mathrm{C}_{56} \mathrm{H}_{67} \mathrm{~N}_{7} \mathrm{NaO}_{10}[\mathrm{M}+\mathrm{Na}]^{+} 1020.4847$, found 1020.4851 .

Diene 10

${ }^{1} \mathrm{H}$ NMR $\left(400 \mathrm{MHz}, \mathrm{CDCl}_{3}\right) \delta$ 7.98-7.61 (m, 2H), 7.49-7.06 (m, 4H), 6.12-5.86 (m, 0.4H, $\mathrm{N} \underline{\mathrm{H}}$ minor rotamer), 5.77 (dddd, $2 \mathrm{H}, \mathrm{J}=7.1,7.1,10.0,17.1 \mathrm{~Hz}), 5.57-5.46(\mathrm{~m}, 1.6 \mathrm{H}, \mathrm{N} \underline{\mathrm{H}}$ major rotamer), $5.15(\mathrm{~d}, 2 \mathrm{H}, \mathrm{J}=17.0), 5.11(\mathrm{~d}, 2 \mathrm{H}, \mathrm{J}=10.1 \mathrm{~Hz}), 4.59-4.36(\mathrm{~m}, 2 \mathrm{H}), 4.18-4.07(\mathrm{~m}, 2 \mathrm{H})$, 4.04-3.81 (m, 4H), 3.35-3.09 (m, 8H), 2.67-2.56 (m, 2H), 2.56-2.43 (m, 2H), 2.41-2.29 (m, $4 \mathrm{H}), 2.19-2.05(\mathrm{~m}, 2 \mathrm{H}), 1.96-1.78(\mathrm{~m}, 2 \mathrm{H}), 1.61-1.47(\mathrm{~m}, 2 \mathrm{H}), 1.59-1.48(\mathrm{~m}, 4 \mathrm{H}),[1.46(\mathrm{~s})$, 1.43 (s), 1.43 (s), $45 \mathrm{H}$ total $\mathrm{ppm} ;{ }^{13} \mathrm{C}$ NMR $\left(100 \mathrm{MHz}, \mathrm{CDCl}_{3}\right) \delta 172.9(\mathrm{C}), 172.0(\mathrm{C}), 171.6$ (C), 169.2(C), 155.9(C), 133.4(CH), 133.3(CH),118.7 $\left(\mathrm{CH}_{2}\right), 118.6\left(\mathrm{CH}_{2}\right), 82.1(\mathrm{C}), 79.8(\mathrm{C})$, 79.5(C), 69.6(C), 54.2( $\left(\mathrm{CH}_{2}\right), 53.6(\mathrm{CH}), 53.4(\mathrm{CH}), 53.2(\mathrm{CH}), 47.0\left(\mathrm{CH}_{2}\right), 43.2\left(\mathrm{CH}_{2}\right), 38.8$ $\left(\mathrm{CH}_{2}\right), 36.2\left(\mathrm{CH}_{2}\right), 32.1\left(\mathrm{CH}_{2}\right), 31.8\left(\mathrm{CH}_{3}\right), 30.9\left(\mathrm{CH}_{3}\right), 29.3\left(\mathrm{CH}_{3}\right), 28.7\left(\mathrm{CH}_{2}\right), 28.5\left(\mathrm{CH}_{3}\right), 28.4$ $\left(\mathrm{CH}_{3}\right), 28.0\left(\mathrm{CH}_{3}\right), 26.5\left(\mathrm{CH}_{2}\right), 25.8\left(\mathrm{CH}_{2}\right) \mathrm{ppm}$; HRMS (ESI) $\mathrm{m} / z$ calcd for $\mathrm{C}_{54} \mathrm{H}_{93} \mathrm{~N}_{9} \mathrm{NaO}_{16}$ $[\mathrm{M}+\mathrm{Na}]^{+} 1146.6633$, found 1146.6594 .

\section{General RCM synthesis of macrocyles 11,12 and 13}

Under an argon atmosphere, a solution of ruthenium catalyst 17 (27-35 mol \%) was added to a dilute solution (2-3 mM) of the diene substrate $\left(\mathbf{8}, 9\right.$, or 10) in $\mathrm{CH}_{2} \mathrm{Cl}_{2}$, which had been degassed by bubbling argon through the mixture for $20-30 \mathrm{~min}$. The resulting solution was gently refluxed for 4 days. The solvent was removed under reduced pressure and the residue was purified by flash chromatography (for 11, two columns were required to remove all traces of the catalyst, 5-10\% $\mathrm{CH}_{3} \mathrm{OH} / \mathrm{CH}_{2} \mathrm{Cl}_{2}$ and then $60-80 \%$ acetone/hexane, for 12, 40-100\% acetone/toluene, and for 13, 5-10\% methanol/ $/ \mathrm{CH}_{2} \mathrm{Cl}_{2}$ ).

\section{Macrocycle 11}

(55\% yield); ${ }^{1} \mathrm{H} \mathrm{NMR}\left(400 \mathrm{MHz}, 5 \% \mathrm{CD}_{3} \mathrm{OD} / \mathrm{CDCl}_{3}\right) \delta$ 7.41-7.23 (m, 10H), 6.61-5.33 (m, $2 \mathrm{H}), 5.18-4.97(\mathrm{~m}, 4 \mathrm{H}), 4.36-4.10(\mathrm{~m}, 2 \mathrm{H}), 3.97-3.65(\mathrm{~m}, 4 \mathrm{H}), 3.36-2.92(\mathrm{~m}, 8 \mathrm{H}), 2.58-2.30$ $(\mathrm{m}, 4 \mathrm{H}), 1.93-1.80(\mathrm{~m}, 2 \mathrm{H}), 1.78-1.56(\mathrm{~m}, 2 \mathrm{H}), 1.54-1.24(\mathrm{~m}, 11 \mathrm{H}) \mathrm{ppm} ;{ }^{13} \mathrm{C} \mathrm{NMR}(100 \mathrm{MHz}$, $\left.5 \% \mathrm{CD}_{3} \mathrm{OD} / \mathrm{CDCl}_{3}\right) \delta 172.7,169.7,156.7,136.2,128.6,128.37,128.35,128.30,128.2,128.1$, 128.0, 127.3, 80.0, 67.2, 55.1, 54.2, 46.8, 42.8, 38.7, 35.5, 35.3, 34.9, 31.8, 29.7, 29.2, 28.4, 27.0, 26.8, 26.31, 26.26, 26.22, 26.12, $25.4 \mathrm{ppm}$; HRMS (ESI) $\mathrm{m} / \mathrm{z}$ calcd for $\mathrm{C}_{40} \mathrm{H}_{55} \mathrm{~N}_{7} \mathrm{NaO}_{10}[\mathrm{M}+\mathrm{Na}]^{+}$816.3908, found 816.3927.

\section{Macrocycle 12}

(75\% yield); ${ }^{1} \mathrm{H}$ NMR (400 MHz, 1:1 $\left.\mathrm{CD}_{3} \mathrm{OD} / \mathrm{CDCl}_{3}\right) \delta 7.78(\mathrm{~d}, 4 \mathrm{H}, \mathrm{J}=7.5 \mathrm{~Hz}), 7.67-7.57(\mathrm{~m}$, $4 \mathrm{H}), 7.40(\mathrm{t}, 4 \mathrm{H}, \mathrm{J}=7.4 \mathrm{~Hz}), 7.31(\mathrm{t}, 4 \mathrm{H}, \mathrm{J}=7.4 \mathrm{~Hz}), 5.58-5.30(\mathrm{~m}, 2 \mathrm{H}), 4.53-4.33(\mathrm{~m}, 4 \mathrm{H})$, 4.26-4.07 (m, 4H), 3.94-3.73 (m, 4H), 3.32-3.30 (m, 8H), 2.55-2.32 (m, 4H), 1.78-1.63 (m, $2 \mathrm{H}), 1.56-1.37$ (m, 13H); LRMS (ESI) $\mathrm{m} / z$ [M+Na] ${ }^{+}$992.4; HRMS (FAB) $\mathrm{m} / z$ calcd for $\mathrm{C}_{54} \mathrm{H}_{63} \mathrm{~N}_{7} \mathrm{NaO}_{10}[\mathrm{M}+\mathrm{Na}]^{+}$992.4534, found 992.4522 .

\section{Macrocycle 13}

(92\% yield); ${ }^{1} \mathrm{H}$ NMR $\left(400 \mathrm{MHz}, \mathrm{CDCl}_{3}\right) \delta$ 8.12-7.64 (M, $\left.2 \mathrm{H}\right)$, 7.56-7.08 (m, 4H), 5.70-5.35 (m, 4H), 4.77-4.25(m, 2H), 4.20-3.59 (m, 6H), 3.44-3.00 (m, 8H), 2.81-2.29 (m, 8H), 2.26-2.00 $(\mathrm{m}, 2 \mathrm{H}), 1.97-1.64(\mathrm{~m}, 4 \mathrm{H}), 1.64-1.29(\mathrm{~m}, 49 \mathrm{H}) \mathrm{ppm} ;{ }^{1} \mathrm{H} \mathrm{NMR}\left(400 \mathrm{MHz}, \mathrm{CD}_{3} \mathrm{OD}\right) \delta$ 5.65-5.47 $(\mathrm{m}, 2 \mathrm{H}), 4.39-4.15(\mathrm{~m}, 2 \mathrm{H}), 4.05-3.94(\mathrm{~m}, 2 \mathrm{H}), 3.93-3.71(\mathrm{~m}, 4 \mathrm{H}), 3.32-3.12(\mathrm{~m}, 8 \mathrm{H}), 2.54-2.32$ $(\mathrm{m}, 8 \mathrm{H}), 2.20-2.02(\mathrm{~m}, 2 \mathrm{H}), 1.91-1.69(\mathrm{~m}, 4 \mathrm{H}), 1.64-1.41(\mathrm{~m}, 4 \mathrm{H}),[1.46(\mathrm{~s}), 1.45(\mathrm{~s}), 1.44(\mathrm{~s})$, $45 \mathrm{H}$ total] ppm; ${ }^{13} \mathrm{C}$ NMR $\left(100 \mathrm{MHz}, \mathrm{CD}_{3} \mathrm{OD}\right) \delta 175.0,174.9,174.1,174.0,173.3,171.5$, $171.4,158.1,157.3,130.0,129.8,82.7,81.4,80.8,80.5,56.5,56.1,55.3,55.2,48.2,46.4,45.8$, 43.6, 39.8, 38.0, 35.8, 35.5, 32.7, 29.6, 28.8, 28.3, 27.4 ppm; LRMS (ESI) $\mathrm{m} / z$ 1118.6 ([M 
$\left.+\mathrm{Na}]^{+}, 100 \%\right), 1134.6\left([\mathrm{M}+\mathrm{K}]^{+}, 25 \%\right)$; HRMS (FAB) $m / z$ calcd for $\mathrm{C}_{52} \mathrm{H}_{89} \mathrm{~N}_{9} \mathrm{Na}_{2} \mathrm{O}_{16}[\mathrm{M}$ $+\mathrm{Na}]^{2+} 570.8106$, found 570.8123 (adding sample and reference together caused singly charged peaks to disappear, therefore, doubly charged $\left(2 \mathrm{Na}^{+}\right)$peaks were used to measure exact mass).

\section{Compound 15}

${ }^{1} \mathrm{H}$ NMR (400 MHz, 10\% $\left.\mathrm{CD}_{3} \mathrm{OD} / \mathrm{CDCl}_{3}\right) \delta 7.76(\mathrm{~d}, 4 \mathrm{H}, \mathrm{J}=7.5 \mathrm{~Hz}), 7.59$ (d, 4H, J=7.3 Hz), $7.40(\mathrm{t}, 4 \mathrm{H}, \mathrm{J}=7.5 \mathrm{~Hz}), 7.31(\mathrm{t}, 4 \mathrm{H}, \mathrm{J}=7.5 \mathrm{~Hz}), 4.54-4.30(\mathrm{~m}, 4 \mathrm{H}), 4.20(\mathrm{t}, 2 \mathrm{H}, \mathrm{J}=6.5 \mathrm{~Hz})$, 4.20-4.07 (m, 2H), 4.01-3.84 (m, 2H), 3.81-3.70 (m,2H), 3.41-2.91 (m, 8H), 1.88-1.57 (m, $6 \mathrm{H}), 1.53-1.25(\mathrm{~m}, 17 \mathrm{H}) \mathrm{ppm} ;{ }^{13} \mathrm{C} \mathrm{NMR}\left(100 \mathrm{MHz}, 10 \% \mathrm{CD}_{3} \mathrm{OD} / \mathrm{CDCl}_{3}\right) \delta 173.0(\mathrm{C}), 169.6$ (C), 156.5(C), 156.4(C), 156.1(C), 155.6(C), 143.5(C), 141.09(C), 141.06(C), 127.6(CH), 126.9(CH), 124.7(CH), 119.8(CH), 79.7(C), 77.2(CH), 66.7 $\left(\mathrm{CH}_{2}\right), 66.5\left(\mathrm{CH}_{2}\right)$, 54.4 $\left(\mathrm{CH}_{2}\right)$, 54.1 $\left(\mathrm{CH}_{2}\right), 47.0(\mathrm{CH}), 46.9(\mathrm{CH}), 46.4\left(\mathrm{CH}_{2}\right), 45.1\left(\mathrm{CH}_{2}\right), 43.4\left(\mathrm{CH}_{2}\right), 42.8\left(\mathrm{CH}_{2}\right), 42.7\left(\mathrm{CH}_{2}\right)$, 38.6 $\left(\mathrm{CH}_{2}\right), 38.5\left(\mathrm{CH}_{2}\right), 36.9\left(\mathrm{CH}_{2}\right), 35.9\left(\mathrm{CH}_{2}\right), 31.1\left(\mathrm{CH}_{2}\right), 30.9\left(\mathrm{CH}_{2}\right), 29.4\left(\mathrm{CH}_{2}\right), 28.1\left(\mathrm{CH}_{3}\right)$, 27.1 $\left(\mathrm{CH}_{2}\right), 25.9\left(\mathrm{CH}_{2}\right), 25.0\left(\mathrm{CH}_{2}\right), 24.0\left(\mathrm{CH}_{2}\right) \mathrm{ppm}$; HRMS (ESI) $\mathrm{m} / \mathrm{z}$ calcd for $\mathrm{C}_{54} \mathrm{H}_{65} \mathrm{~N}_{7} \mathrm{NaO}_{10}[\mathrm{M}+\mathrm{Na}]^{+}$994.4691, found 994.4680 .

\section{Compound 16}

${ }^{1} \mathrm{H} \mathrm{NMR}\left(400 \mathrm{MHz}\right.$, ca. $\left.1: 1 \mathrm{CDCl}_{3} / \mathrm{CD}_{3} \mathrm{OD}\right) \delta 4.42-4.09(\mathrm{~m}, 2 \mathrm{H}), 4.05-3.66(\mathrm{~m}, 6 \mathrm{H}), 3.35-3.00$ $(\mathrm{m}, 8 \mathrm{H}), 2.44-2.22(\mathrm{~m}, 4 \mathrm{H}), 2.18-1.97(\mathrm{~m}, 2 \mathrm{H}), 1.95-1.62(\mathrm{~m}, 8 \mathrm{H}), 1.90-1.65(\mathrm{~m}, 8 \mathrm{H}), 1.62-1.15$ $(\mathrm{m}, 45 \mathrm{H}) \mathrm{ppm} ;{ }^{13} \mathrm{C} \mathrm{NMR}\left(100 \mathrm{MHz}\right.$, ca. $\left.1: 1 \mathrm{CD}_{3} \mathrm{OD} / \mathrm{CDCl}_{3}\right) \delta 174.3,174.2,171.0,157.3$, 157.0, 156.9, 156.8, 82.6, 81.1, 80.4, 80.3, 78.9, 56.0, 54.7, 54.3, 47.8, 46.2, 45.1, 43.6, 39.6, $39.5,37.8,37.3,36.1,35.5,32.4,31.9,31.5,29.3,28.9,28.8,28.4,27.8,27.6,27.4,26.9,26.9$, 26.8, 26.3, 25.9, 25.2, 24.6 ppm; LRMS (ESI) $\mathrm{m} / z$ 1120.6 [M+Na] ${ }^{+}$; HRMS (ESI) $\mathrm{m} / z$ calcd for $\mathrm{C}_{52} \mathrm{H}_{91} \mathrm{~N}_{9} \mathrm{NaO}_{16}[\mathrm{M}+\mathrm{Na}]{ }^{+} 1120.6476$, found 1120.6475 .

\section{Supplementary Material}

Refer to Web version on PubMed Central for supplementary material.

\section{Acknowledgements}

We thank Professor Christopher Walsh and Kari Nadeau (Department of Biological Chemistry and Molecular Pharmacology, Harvard Medical School) for providing the SG5 E. coli strain containing TR expression vector pIBITczTR. This work was supported by a grant from the National Institutes of Health (1 R15 AI053113-01). Acknowledgment is made to the donors of The Petroleum Research Fund, administered by the American Chemical Society, for partial support of this research. Student stipends for J. A. C., M. D. S., and L. K. E. were provided by a grant from the Howard Hughes Medical Institute.

\section{References}

1. Kreier, JP.; Baker, JR., editors. Parasitic Protozoa. Allen \& Unwin; Winchester, MA: 1987. p. 43-89.

2. Fairlamb AH, Blackburn P, Ulrich P, Chait BT, Cerami A. Science 1985;227:1485-1487. [PubMed: 3883489]

3. Fairlamb AH, Cerami A. Annu Rev Microbiol 1992;46:695-729. [PubMed: 1444271]

4. Krauth-Siegel RL, Bauer H, Schirmer RH. Angew Chem Int Ed 2005;44:690-715.and references cited therein

5. (a) Tovar J, Wilkinson S, Mottram JC, Fairlamb Alan H. Mol Microbiol 1998;29:653-660. [PubMed: 9720880] (b) Krieger S, Schwarz W, Ariyanayagam MR, Fairlamb AH, Krauth-Siegel RL, Clayton CH. Mol Microbiol 2000;35:542-552. [PubMed: 10672177]

6. Bond CS, Zhang Y, Berriman M, Cunningham ML, Fairlamb AH, Hunter WN. Structure (London) 1999;7:81-89.and references cited therein

7. Ghisla S, Massey V. Eur J Biochem 1989;181:1-17. [PubMed: 2653819] 
8. (a) Shames SL, Fairlamb AH, Cerami A, Walsh CT. Biochemistry 1986;25:3519-3526. [PubMed: 3718941] (b) Henderson GB, Fairlamb AH, Ulrich P, Cerami A. Biochemistry 1987;26:3023-3027. [PubMed: 3607007]

9. For recent reviews, see: (a) O'Sullivan MC. Curr Med Chem: Anti-Infective Agents 2005;4:355-378. (b) Krauth-Siegel RL, Inhoff O. Parasitol Res 2003;90(Suppl 2):S77-S85. [PubMed: 12709793] (c) Austin SE, Khan MOF, Douglas KT. Drug Des Discov 1999;16:5-23. [PubMed: 10466053]

10. (a) El-Waer AF, Smith K, McKie JH, Benson T, Fairlamb AH, Douglas KT. Biochim Biopys Acta 1993;1203:93-98. (b) Tromelin A, Moutiez M, Meziane-Cherif D, Aumercier M, Tartar A, Sergheraert C. Bioorg Med Chem Lett 1993;3:1971-1976. (c) Garrard EA, Borman EC, Cook BN, Pike EJ, Alberg DG. Org Letters 2000;2:3639-3642.

11. Scholl M, Ding S, Lee CW, Grubbs RH. Org Letters 1999;1:953-956.

12. For recent reviews, see: (a) Prunet J. Angew Chem Int Ed 2003;42:2826-2830. (b) Van de Weghe P, Eustache J. Curr Top Med Chem 2005;5:1495-1519. [PubMed: 16378489]

13. Henderson GB, Glushka J, Cowburn D, Cerami A. J Chem Soc Perkin Trans 1 1990:911-914.

14. (a) Chantrapromma K, McManis JS, Ganem B. Tetrahedron Lett 1980:2475-2476. (b) Ganem B. Acc Chem Res 1982;15:290-298.

15 . We previously reported selective acylation of a primary over a secondary amine with succinimide esters; see reference ${ }^{10 \mathrm{c}}$. Selective trifluoroacylation of the primary amino groups of spermidine and related polyamines has also been reported; see: O'Sullivan MC, Dalrymple DM. Tetrahedron Lett 1995;36:3451-3452.

16. PyBop = benzotriazole-1-yloxytrispyrrolidinophosphonium hexafluorophosphate. Coste J, LeNguyen D, Castro B. Tetrahedron Lett 1990;31:205-208.

17. DEPBT = 3-(diethoxyphosphoryloxy)-1,2,3-benzotriazin-4(3H)-one. Li H, Jiang X, Ye Y-h, Fan C, Romoff T, Goodman M. Org Letters 1999;1:91-93.

18. Sullivan FX, Walsh CT. Mol Biochem Parisitol 1991;44:145-148.

19. El-Waer A, Douglas KT, Smith K, Fairlamb AH. Anal Biochem 1991;198:212-216. [PubMed: 1789428]

20. Sullivan FX, Shames SL, Walsh CT. Biochemistry 1989;28:4986-4992. [PubMed: 2669965]

21. For 2, four different inhibitor concentrations were used, varied from $0.70 \mathrm{mM}$ to $10.3 \mathrm{mM}$. For $\mathbf{3}$, three different inhibitor concentrations were examined, ranging from $228 \mu \mathrm{M}$ to $912 \mu \mathrm{M}$. For all inhibitors, each inhibitor concentration was examined over a range of four substrate concentrations, with all assays performed in at least duplicate. See the Supporting Information for full experimental details.

22. Douglas and coworkers used the enzyme from $C$. fasciculata in their study, which accounts for the difference in the $\mathrm{Km}$ value they report for $\mathbf{1 8}(52 \mu \mathrm{M})$, compared to our determination of this parameter using the T. cruzi enzyme $(6.7 \mu \mathrm{M})$.

23. Khan MOF, Austin SE, Chan C, Yin H, Marks D, Vaghjiani SN, Kendrick H, Yardley V, Croft SL, Douglas KT. J Med Chem 2000;43:3148-3156. [PubMed: 10956223]

24. Saravanamuthu A, Vickers TJ, Bond CS, Peterson MR, Hunter WH, Fairlamb AH. J Biol Chem 2004;279:29493-29500. [PubMed: 15102853]

25. Garforth J, Yin H, McKie JH, Douglas KT, Fairlamb AH. J Enzyme Inhib 1997;12:161-173. [PubMed: 9314113] 

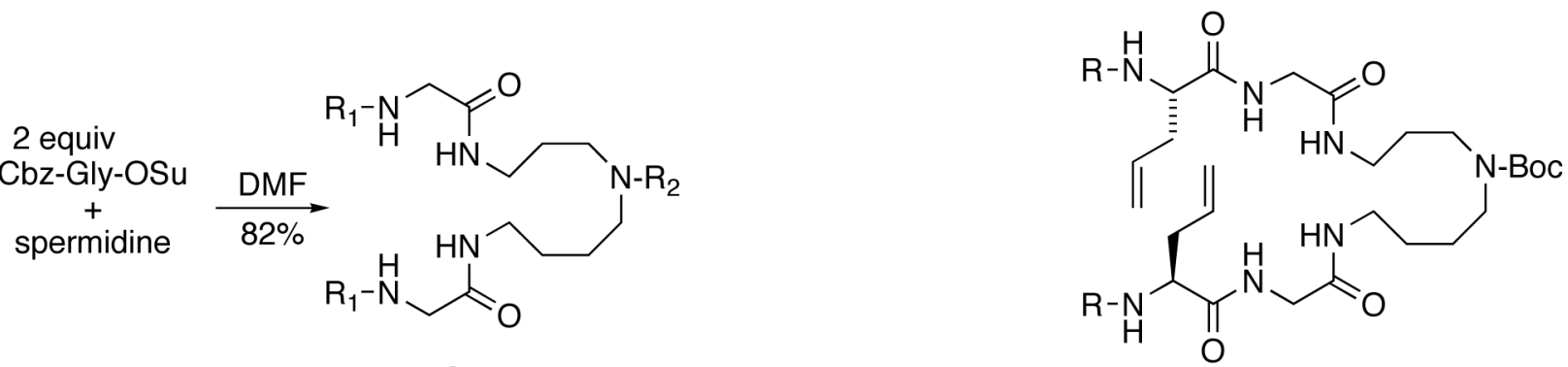

$$
\begin{aligned}
(\text { Boc })_{2} \mathrm{O}, 98 \% \\
\mathrm{H}_{2}, \mathrm{Pd} / \mathrm{C}
\end{aligned}\left\{\begin{array}{l}
5: \mathrm{R}_{1}=\mathrm{Cbz}, \mathrm{R}_{2}=\mathrm{H} \\
6: \mathrm{R}_{1}=\mathrm{Cbz}, \mathrm{R}_{2}=\mathrm{Boc}
\end{array}\right.
$$

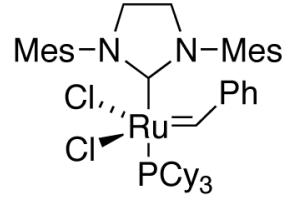

17
1. $\mathrm{Et}_{2} \mathrm{NH}, \mathrm{CH}_{3} \mathrm{CN}$

2. 2 equiv Boc-Glu- $\alpha-t-B u$, DEPBT

10: $R=N-B o c-\alpha-t-B u-\gamma-G l u$

27-35 mol\% 17,

$\mathrm{CH}_{2} \mathrm{Cl}_{2}$, reflux, 4 days

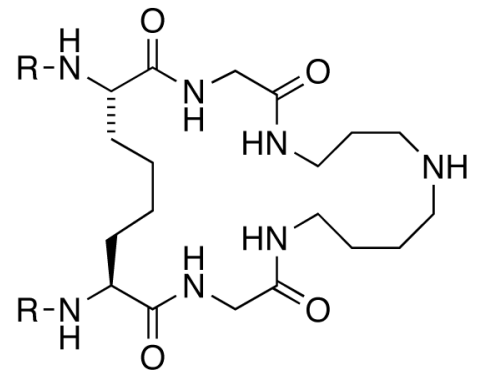

3: $\mathrm{R}=\mathrm{Cbz}, \mathbf{8 4} \%$ from $\mathbf{1 1}$

4: $R=F m o c, 91 \%$

2: $R=\gamma$-Glu, $77 \%$

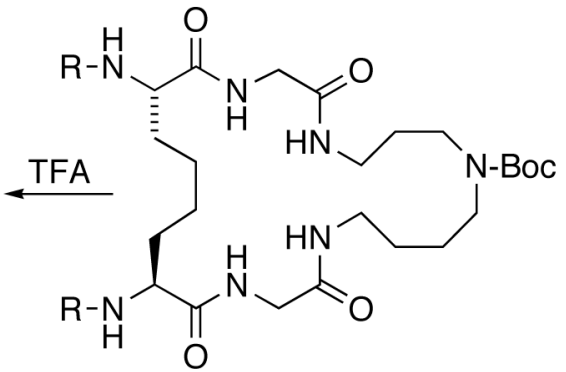<smiles></smiles>

Scheme 1.

Synthesis of Trypanothione Analogues 
Table 1

Inhibition of T. cruzi $\mathrm{TR}^{a}$

\begin{tabular}{ccc}
\hline Entry & Inhibitor & $\mathbf{K}_{\mathbf{i}}(\boldsymbol{\mu} \mathbf{M})$ \\
\hline 1 & $\mathbf{2}$ & $826 \pm 76$ \\
2 & $\mathbf{3}$ & $145 \pm 24$ \\
3 & $\mathbf{4}$ & $16 \pm 2$ \\
\hline
\end{tabular}

${ }^{a}$ Our determination of $\mathrm{K}_{\mathrm{m}}$ for assay substrate $\mathbf{1 8}$ was $6.7 \mu \mathrm{M}$. 